\title{
The regulatory control over radiation sources: the Brazilian experience and some lessons learned from industrial applications
}

\author{
E. L. C. Costa ${ }^{a}$ J. D. R. L. Gomes ${ }^{a}$, R. S. Gomes ${ }^{a}$, M. L. L. Costa ${ }^{a}$ Z. D. Thomé \\ a Comissão Nacional de Energia Nuclear / Diretoria de Radioproteção e Segurança Nuclear, 22290-901, Rio de Janeiro, \\ RJ, Brazil \\ ${ }^{\mathrm{b}}$ Instituto Militar de Engenharia / Seção de Engenharia Nuclear, 22291-270 Rio de Janeiro, RJ, Brazil
} evaldo@cnen.gov.br

\begin{abstract}
This study gives an overview of the activities of the National Commission of Nuclear Energy (CNEN), as the Brazilian nuclear regulatory authority. These activities are described, especially those related to management of orphan sources and radioactive material in scrap metal considering the actions already put into place by CNEN during the licensing and controlling of radioactive sources in the industry and other facilities. In Brazil, there is not yet an effective system for controlling the scrap metal and recycling industry, thus a coordinated approach to achieve a harmonized and effective response with the involvement of third parties is needed, especially the metal industries and ores facilities. These practices call for stringent regulatory control, in order to reduce the occurrence of orphan sources, and consequently, radioactive material appearing in scrap metal. Some challenges of managing the national radiation sources register systems will be discussed, in order to cover effectively all the radiation source history (in a 'from the cradle to the grave' basis), and the dynamic maintenance and update of these data. The main industrial applications considered in this work are those dealing with constant movement of sources all over the country, with geographical issues to be considered in the managing and controlling actions, such as gammagraphy and well-logging. This study aims to identify and promote good practices to prevent inadvertent diversion of radioactive material, taking into account existing international recommendations and some lessons learned in national level.
\end{abstract}

Keywords: Regulatory control, Radioactive sources, Industrial Applications

ISSN: 2319-0612

Accept Submission 2018-12-12 


\section{INTRODUCTION}

According the International Atomic Energy Agency (IAEA), an orphan source is a radioactive source which is not under regulatory control, either because it has never been under regulatory control or because it has been abandoned, lost, misplaced, stolen or otherwise transferred without proper authorization. A vulnerable source is a radioactive source for which the control is inadequate to provide assurance of long term safety and security, such that it could be relatively easily be acquired by unauthorized persons. A radioactive source that is no longer in use or not intended to be used, for the practice for which an authorization has been granted, is termed as disused [1].

Although the vast majority of radioactive sources used around the world are managed safely and securely, the occurrence of accidents involving orphan sources in 90s, as well as the attack of September 11th 2001, led IAEA to make efforts for the establishment of the Code of Conduct on the Safety and Security of Radioactive Sources [2] [3]. The main purposes of the Code are the strengthen the security, management and control of radioactive sources from a global cradle-to-grave perspective, including the manufacture, distribution, licensing, export, import, recycle, disassembly and disposal.

Nevertheless, the possibility of orphan sources being present in scrap metal or its use for malicious purposes remains, thus these concerns have led many States to address issues relating to the control of radioactive sources and to initiate a programme of work on the safety and security of sources. On the other hand, metal recycling has become an important industrial activity through the world; it enables us to preserve natural resources while requiring less energy to process in comparison with the manufacture using virgin raw materials, thus the process is environmentally beneficial [4] [5]. However, there have been several accidents involving orphan radioactive sources or other radioactive material that were inadvertently collected as scrap metal that was destined for recycling. An accident with serious consequences regarding the people and environment may occur if the radioactive material is not detected at the metal recycling and production facility. Thus, this issue calls for stringent regulatory control, in order to reduce the occurrence of orphan sources, and consequently, radioactive material appearing in scrap metal [6]. 
The purpose of this study is to identify and promote good practices to prevent inadvertent diversion of radioactive material, taking into account existing international recommendations and some lessons learned in national level.

Additionally, it will be given an overview of the activities of the CNEN, as the Brazilian nuclear regulatory authority on dealing with radiation source controlling, focusing the proper management of disused sources. These actions aim the goal of reducing the probability of orphan sources and consequently the occurrences of scrap metal in the industry.

Finally, it will be proposed a methodology for establishment of a strategy for recovery the control over orphan sources and improving control over disused sources focusing those which may be categorized as vulnerable sources.

\section{GENERAL ACTIVITIES OF CNEN}

The National Commission of Nuclear Energy (CNEN) is a federal autarchy linked to the Ministry of Science, Technology, Innovations and Communications. The range of its responsibilities is very broad, including regulating, licensing, controlling and inspecting as well as research. Its mission is 'To facilitate the secure and peaceful use of nuclear energy, to develop and make available nuclear technology for the benefit of the population' [6].

CNEN has branches throughout the country: in addition to the board of directors that administers the regulatory body itself, it is organized with two technical directorates, the Directorate of Radiation Protection and Nuclear Safety (DRS) and the Directorate of Research and Development (DPD).

The DRS coordinates, regulates and oversees the execution of the activities of licensing and inspection of nuclear and radioactive facilities, inspection of mining and benefiting industries of ores containing uranium and thorium, nuclear safety, radiation protection, safeguards, physical protection, controlling of nuclear and radioactive materials and ores of strategic interest and certification of professionals in these fields.

Although there is no direct responsibility over the controlling of scrap metal and recycling industry, DRS has a direct responsibility for the control of orphan sources through licensing and controlling nuclear and radioactive facilities and mining industries. 
The DPD plans, orientates and coordinates the execution of the activities of research, development and nuclear technological and ionizing radiation applications, and is responsible for education and training concerning technical and scientific specializations and graduation in the nuclear field.

Although it is not directly concerned with scrap metal, the DPD is involved in cooperation work with DRS, and, for example, it contributes to the nonproliferation of orphan sources by placing disused radioactive sources in secure storage facilities in the DPD institutes and regional centres, namely the Institute of Energetic and Nuclear Research (IPEN), the Institute of Nuclear Engineering (IEN), the Center of Nuclear Technology Development (CDTN), the Regional Center of Northeast (CRCN-NE) and the Regional Center of Mid-West (CRCN-CO).

In relation to licensing, the emphasis is on stringent processes in the safety assessment phase for the major risk facilities, completed by inspection and auditing actions. In exercising control, all the available actions and tools for controlling radioactive sources are applied, be it to those produced and distributed throughout the national territory, or to those imported from foreign countries [7] [8] [9]. The Integrated System of Foreign Trade (SISCOMEX) is a tool used in the control of Brazilian foreign trade, and, in relation to the interests of CNEN, it is applied to the import and export process of products containing radioactive material, strategic raw material for the country or radiation generator equipment [10].

This system is used by several Brazilian governmental authorities, among them, CNEN, through partnership with the institutions responsible for the management of this system, namely: The Secretariat of Foreign Trade (SECEX), linked to the Ministry of Development, Industry and Foreign Trade; the Secretariat of Federal Revenue of Brazil and the Central Bank of Brazil, both linked to the Ministry of Economy.

Additionally, CNEN has a database registering all radioactive sources used throughout the country. This system makes it easier to identify potential orphan sources when a notification is received through the system of response to radiological events [6].

These actions of licensing and controlling together establish regulatory control over practices and sources, decreasing the probability of sources becoming orphaned and consequently, appearing in scrap metal. 


\section{REGULATORY RESPONSIBILITIES AND STAKEHOLDERS INVOLVEMENT}

The Brazilian regulatory body, through their various departments, is responsible for the licensing of radioactive materials applied to medicine, research, industry, commerce and service providers, as well as for the control of the sources related to the associated practices.

In relation to licensing, the emphasis is on stringent processes in the safety assessment phase for the major risk facilities, completed by inspection and auditing actions; in exercising control, all the available actions and tools for controlling radioactive sources are applied, be it to those produced and distributed throughout the national territory, or to those imported from foreign countries.

On the other hand, these actions should be in accordance with IAEA recommendations [4], with respect on issue of vulnerable and orphan sources in metal recycling and production industries, which stands that the regulatory body is responsible by:

- Maintaining oversight of radiation security within these industries. In order to do this, it should establish and maintain a list of the metal recycling and production facilities;

- Working towards building a relationship with the industries in order to ensure effective cooperation in the event of an incident or emergency involving radioactive material;

- Participating in any initiative of the metal recycling and production industries to develop guides, agreements or protocols relating to the protection of workers, members of the public and the environment from hazards associated with radioactive material that may be present in scrap metal;

- Disseminating information, in cooperation with the metal recycling and production industries, to facilities regarding the injuries that can be caused by radioactive material in scrap metal, particularly dangerous sources, the actions to be taken in the event of the discovery of radioactive material, and the lessons learned from past events involving the presence of radioactive material in the metal recycling and production industries;

- Development of policies and strategies for the control of scrap metal, metal products or wastes that might contain radioactive material, in cooperation with other relevant national authorities, including customs and border authorities; 
- Establishment of arrangements, cooperation with the competent authority, in the response to incidents and emergencies involving the discovery of radioactive material in the metal recycling and production industries;

- Assisting in regaining physical control over any radioactive material discovered in scrap metal. It should also ensure that any radioactive material recovered is appropriately stored pending its removal to a radioactive waste storage or disposal facility authorized to handle the material;

- Undertaking its own investigation or should assist in any investigation into any incident involving the detection of radioactive material at a metal recycling and production facility, to determine the possible causes and any lessons to be learned and whether any additional controls may need to be implemented.

Regarding the metal recycling and production industries, the operator is responsible for the health and safety of its workers and any other persons who may be affected by its actions and activities, including members of the public; also, the organizations and associations representing this sector should support the development of industrial standards for metal recycling and production [4] [5].

So, it is clear that CNEN should foster the debate on regulatory control of radioactive sources, focusing the disused sources, with special attention to the vulnerable sources, in order to minimize the probability of existence of orphan sources and its insertion on the scrap metal and recycling industry. This initiative should involve its licensees and other stakeholders who might take part in this matter, in order to harmonize national regulation and procedures with the IAEA recommendations [2] [4] [5].

\section{SEALED RADIOACTIVE SOURCES IN INDUSTRIAL FACILITIES}

In industrial applications, the area likely to cause most concern is industrial gamma radiography. In Brazil, mainly Iridium-192 and Selenium-75 sources are used for on-site facilities and Cobalt-60 for enclosed facilities. The service providers, around 20 companies, together have a total inventory of about a hundred sources spread throughout the country. 
For this reason, stringent control is exerted over these companies, through the inclusion of special conditions in their authorizations for the operation of these sources, specifying the maximum capacity of the sources commensurate with the radiation protection system provided in the operations (calibrated survey meters and qualified personnel in adequate numbers proportional to the source inventory) and the licensed capacity of the storage for the sources when not in use [11]. These companies are required to notify CNEN monthly regarding all the contracts they receive to provide industrial radiography services at third parties' premises, with all information about these facilities included.

There may be critical situations when these sources are to be used in inhabited areas and on public roads, as in the case of oil and gas pipelines. In these cases, the special conditions in the authorizations are even more constraining and specific for each individual service; the related notifications to the regulatory authority are similar to the conventional operations using gamma radiography sources, but with specific information to be provided about these services.

In oil and gas exploration, there are more than ten operational bases belonging to the three biggest multinationals in this field; in addition, there are some other companies of medium and small size, in the fields of well logging and cementation, making use of Cesium-137 and neutron sources, as well as low activity sources used for calibration of logging tools [6] [12].

In the nuclear gauging field, there are several hundred of nucleonic gauges in use by at least 500 industrial facilities. Some are being used in severe work conditions, such as exposure to high temperatures which could produce accidents involving the melting of the source shield thereby causing the production of contaminated material and a potential source of contaminated scrap metal — if there is not stringent control over the facility [6].

Industrial irradiation facilities, although belonging to the major risk category, generally do not cause a great deal of concern when compared to the others. This is because of the defence in depth and safety culture approaches adopted when designing, constructing and operating these facilities and because of the care given by the regulatory body in licensing them [6] [7] [8] [9].

During decommissioning, it is necessary to be sure that all activities are carried out under proper control until the sources of Cobalt-60 have been repatriated or taken to an intermediate storage facility accepted by CNEN prior to their final destination. Otherwise, the orphan sources or worse, radioactive 
scrap metal, coming from the mismanagement of this decommissioning could lead to an accident of enormous proportions [5] [9].

\section{LESSONS LEARNED FROM INDUSTRIAL APPLICATIONS}

\section{Bankruptcy of Well Logging Facility}

Considering the problems in the oil and gas exploration and drilling area that affected Brazil from 2015 onwards, a well logging service company no more received the financial support of its multinational matrix and went bankrupt. The inventory of radioactive sources in the facility was of 33 radioactive sources of Cesium-137 and Americium-Beryllium. With its bankruptcy, the company did not have the financial means to return the radioactive sources back to the country of origin or transfer it to disposal at national repository.

The regulatory body became aware of the situation when it was activated by the Public Prosecution. The regulatory body covered the whole cost of transporting and depositing the sources, including all the logistics of the operation. It should be emphasized that the operation had delay of some months due to scarcity of funds. During this time, there was a dangerous decrease in the security of the sources, making them vulnerable during this period.

In this event, it was clear the necessity of creation of financial arrangements for dealing with the response to and the consequences for emergency and immediate actions related to disused radioactive sources, especially in cases of bankruptcy of facilities or orphan sources.

\section{Loss of Control of Cesium-137 Radiometric Gauge Source}

Another situation to be emphasized is about a radioactive source temporarily lost in the radiometric gauge application. A certain industrial facility in the mining area using dozens of radioactive sources of Cesium-137 in order to control the ore beneficiation process.

In March 2017, the control room of this facility recorded the loss of the signal from a radiometric gauge, installed in the industrial plant for density measurement. The operation teams in control room were not alerted immediately to the situation. 
Only two days later the maintenance team was sent to perform a check on the equipment. It was observed that the shutter was not present and there was no emission of radiation. At this moment an alert was issued at the facility and efforts were made to localize the radioactive source through extensive monitoring of the facility. The source was found about 10 hours after the alarm, in a metal scrap bin. The source was recovered and stored securely in the source storage location of the facility. The facility has pointed out that there could have been an intentional act on the event, however, the preliminary report performed by regulatory body pointed the failures in the operational procedure that may have not followed the safety recommendations of the equipment manufacturer regarding the locking of the radiometric gauge source after the movement of the shutter.

Disabling two of the three locking systems, a favorable situation has been created, due to vibrations and mechanical shocks, for the fall of the source in the floor, which was collected along other scraps inadvertently by the cleaner team and placed in the metal scrap bin. In this event the source was finally recovered; however, it became evident that the lack of safety culture of radioactive facilities may lead to occurrences of radioactive material in scrap metal.

\section{PLAN OF ACTION FOR EMPOWERMENT OF CONTROLLING OF RADIOACTIVE SOURCES}

The recommendations proposed by IAEA, with respect on issue of vulnerable and orphan sources in metal recycling and production industries, are partially complied by CNEN. However, minor adjustments should be done in some procedures and tools, as well as there are new actions to be performed by CNEN in not yet covered fields. These two cases are discussed below in detail.

The Brazil has the basic requirements to provide the safety and security of sources with the purpose to maintain the continuous control throughout their life cycle, such as the national registry of sources, the import and export control for all radioactive sources and the emergency preparedness and response plan.

However, it should be emphasized that it is lacking to CNEN the elaboration of a methodology for establishing a national strategy for regaining control over orphan sources and improving control over 
vulnerable sources in order to meet the safety and security requirements established in the IAEA safety standards SSG-19 [5].

\subsection{Enhancement of Actions Already Accomplished}

Taking into account the IAEA recommendations, this section presents proposals for improvements in procedures and tools already established in Brazil, such as: national registry of radioactive sources and system of foreign trade.

\section{National Registry of Sources}

CNEN has a database registering sources and practices involving radioactive material, regardless of the radioactive source categorization, as part of regulatory information. However, some improvement is need to maintain the efficiency and effectivity of these tool [6].

The reliability of the data available in the CNEN database should be continually reviewed, mainly taking into account the oldest facilities which did not have their decommissioning process formally concluded, according CNEN standards [9]. The lack of information on the decommissioning of these facilities may imply the proliferation of orphan sources throughout the country and eventual insertion on scrap metal and the recycling industries.

There are some inconsistencies on the traceability of sources during their life cycle, mainly due to the nonexistence of cross checking routines between interdependent systems used for the licensing of facilities and the control of radioactive sources.

Besides, there is also the need of upgrading the database system of the control of sources in order to include some information concerning the IAEA categorization of radioactive sources on safety and security aspects [13] [14].

Despite the need of some improvements in the database, the main concern is that there is a lack of constant maintaining and updating of the database for an effective control of radioactive sources, thus organizational arrangements should be done to remedy this deficiency.

\section{The Integrated Foreign Trade System - SISCOMEX}

The Brazilian control system for the import and export of radioactive sources is based on a joint action between the CNEN and Brazilian Customs. Any imported or exported product is registered using an 
on-line system for control of foreign trade — the integrated foreign trade system SISCOMEX. The materials or equipment registered in SISCOMEX are classified following the Mercosul Common Nomenclature (NCM) [10].

Several governmental organizations require the blockage by default of the import or export of a number of products described by specific NCM items. The import or export operation of these products is authorized by Brazilian Customs only after the end of a parallel authorization process in different regulatory agencies, which has requested the blockage of the specific NCM item and is able to unblock, on-line, the import or export operation in SISCOMEX.

The CNEN then requests the blockage of a number of NCM items, which cover all radioactive (and nuclear) sources and equipment capable of producing ionizing radiation [10]. Thus, the consent of different regulatory authorities is distributed accordingly by the system, considering the respective attributions of each goods receipt under appraisal.

It should be emphasized that the possibility of entry of radioactive material without the consent of CNEN is directly linked to the capability to blockade all possible NCMs that may be inadvertently used during foreign trade, in this way a constant evaluation of the nomenclatures must be carried out. To exemplify the need for this review, there was the import of a vehicle (truck) equipped with an electron accelerator for cargo scanning. The equipment was registered by the buyer in the SISCOMEX using the NCM nomenclature regarding to one special vehicle. In this case, as the NCM nomenclature was not blocked by CNEN, the equipment entered the country without the proper consent by CNEN.

\subsection{New Actions to Be Established}

In this section are presented the IAEA recommendations, with respect on issue of vulnerable and orphan sources in metal recycling and production industries, which are not performed by CNEN and need to be established, taking into account its responsibility as the national nuclear regulatory body, such as: enhancement of regulatory framework and oversight, as well as some strategies for identification of orphan sources [4] [5]. 


\section{Enhancement of Regulatory Framework and Oversight}

The CNEN provides regulatory oversight over industrial facilities which use radioactive sources, focusing mainly the safety aspects. However, in agreement with international recommendations, a more comprehensive view over security aspects is needed [2] [13].

In recent years CNEN has made an effort to update their standards. It is planned to 2018 the approval of two important documents: a specific for security of radioactive sources and another for well logging activities. However, in the industrial area, other standards should be elaborated, such as for irradiation facilities, radiometric gauges and radiotracers.

\section{Identifying the Orphan Source Sensitive Facilities}

CNEN do not maintain any list of metal recycling facilities. It should be emphasized that the preparation of such list is necessary for the regulatory body to be proactive in identifying problems involving vulnerable or orphan sources in these industries.

It should be developed a list comprising the so called orphan source sensitive facilities (OSSF), mainly the metal recycling and production industries. It is an important step in the national strategy to regain control over orphan sources. This list should be established with the stakeholders, who should be identified accordingly [4] [5] [15].

\section{Guidance to Radiation Monitoring}

OSSFs have a higher probability of being confronted with an orphan source than others facilities. Consequently, some countries promote an enhancement of regulatory framework with those facilities have to comply with the obligation of installing of portal monitors [15].

In Brazil, the installation of portal monitors is not compulsory. A few steel and metal businesses of large and medium size have already installed some radiation detection systems in their facilities, with the goal of detecting the presence of items contaminated by radioactive materials, or even the presence of orphan sources, among the metallic scrap received as raw material [6]. Despite these voluntary acts of some of the steel and metal industries, there are still no requirements or even guidance issued by CNEN to orientate these industries. 
It should be noted that the introduction of portal monitors brings issues and doubts regarding the financial responsibility for treatment associated with radioactive materials and sources discovered in their facilities. In the national regulatory framework is not clear that, when a radioactive source is found and its owner cannot be identified, the management and treatment costs for this orphan source should be paid by the regulatory body and not by the finder. Such fact can compromise the installation of new portals. It important to highlight that this matter is an open issue being discussed in several countries [15] [16].

\section{Promotion and Communication}

Efforts must be made by CNEN to build a relationship with the industries in order to ensure cooperation to disseminate information, to develop guides, agreements or protocols involving the presence of radioactive material in the metal recycling and production industries.

In the medium term, there are good prospects for better coordination provided that the right partnerships can be found. Potential partners are some secretariats belonging to Brazilian Ministries, which can help in many ways in introducing the philosophy of security and safety in the scrap metal and recycling industry, by promoting the introduction of radiation detection systems at strategic points, like ports and airports, in large industries in the steel and metal business; by helping to identify associations or any kind of organization which brings together medium and small sized recycling businesses, and also the scrapyards [6].

\section{Response and Recovery}

The licensed facility is the responsible for the cost of recovery or disposal of the radioactive source. If the facility is in bankruptcy or the owner cannot be found as in case of orphan sources, the regulatory body will provide the safe disposal and will cover the whole cost of transporting and depositing the sources, including all the logistics of the operation. Thus, financial arrangements should be available for dealing with the response to and the consequences for emergency and immediate actions related to these cases.

This issue was discussed in the section 5, dealing with the necessity of creation of financial arrangements for emergency and immediate actions related to disused radioactive sources or orphan sources. The creation of this fund is still under study. 


\section{CONCLUSIONS}

Despite the fact that Brazil does not have specific arrangements for managing the problem of radioactive material in the scrap metal and recycling industry, it is clear that CNEN is in control over the sources and practices existing in Brazil. It does this by stringent licensing, inspections and audits, completed by the control of commerce, including domestic and foreign trade.

Brazil has a good infrastructure for collecting of potential orphan sources. Sources no longer in use are then stored at institutes belonging to CNEN. In addition, there is a system of response to radiological emergencies which contributes to the finding of potential orphan sources, contaminated material and occasional scrap metals. Stringent measures are employed in decommissioning radioactive facilities, especially those of major risk classification.

Nevertheless, in addition to all of these actions, it is recognized that more can be done by developing a coordinated mechanism in Brazil for management of orphan sources and radioactive material in scrap metal.

In this study was pointed out the national challenge in implementing reliable tools, procedures and regulation in order to constituting a plan of action, involving all potential stakeholders, towards an effective controlling of radioactive sources, with special attention to disused and vulnerable sources and strategies to the proper management of orphan sources as well as to the scrap metal and the recycling industries.

\section{REFERENCES}

1. IAEA - International Atomic Energy Agency. IAEA Safety Glossary: Terminology Used in Nuclear, Radiation, Radioactive Waste and Transport Safety, 2016 Edition, IAEA, Vienna, 2007. 219p.

2. IAEA - International Atomic Energy Agency. Code of Conduct on the Safety and Security of Radioactive Sources. Vienna, 2004.122p. 
3. JONES, C. G. Consequence Management of Malevolent Use of Radioactive Material Strategies for Enhancing Security of Radioactive Materials. IRPA12 Refresh Course. Buenos Aires, 2008. 21p.

4. IAEA - International Atomic Energy Agency. Control of Orphan Sources and Other Radioactive Material in the Metal Recycling and Production Industries. IAEA SSG-17, Vienna, 2012.104p.

5. IAEA - International Atomic Energy Agency. National Strategy for Regaining Control over Orphan Sources and Improving Control over Vulnerable Sources. IAEA SSG-19, Vienna, 2011. 120p.

6. COSTA, E. L. C. Assignment of Responsibilities in the Management of Scrap Metal in Brazil. In: International Conference on Control and Management of Radioactive Material Inadvertently Incorporated into Scrap Metal, Tarragona, 2011. p.127-135.

7. CNEN - Comissão Nacional de Energia Nuclear. Diretrizes Básicas de Radioproteção. CNEN-NN-3.01, CNEN, Rio de Janeiro, 2014. 22p.

8. CNEN - Comissão Nacional de Energia Nuclear. Serviços de Radioproteção. CNEN-NE3.02, CNEN, Rio de Janeiro, 1988. 17p.

9. CNEN - Comissão Nacional de Energia Nuclear. Licenciamento de Instalações Radiativas. CNEN-NN-6.02, CNEN, Rio de Janeiro, 2014. 14p.

10. GUTTERRES, R. F.; SOUZA, A. L.; MARÉCHAL, M. H. Impact of The Code of Conduct on The Safety and Security of Radioactive Sources on The Brazilian Control System for Import and Export of Radioactive Sources. In: Safety and Security of Radioactive Sources: Towards a Global System for The Continuous Control of Sources Throughout Their Life Cycle, Bordeaux, 2005. p.385-390

11. CNEN - Comissão Nacional de Energia Nuclear. Serviços de Radiografia Industrial. CNEN-NN-6.04, CNEN, Rio de Janeiro, 2014. 16p.

12. GOMES, R. S; LOPES GOMES, J. D.; COSTA M. L.; MIRANDA, M. V. Relevant aspects of radiation protection in oil and gas well logging. Journal of Radiological Protection. 33(4) p. 839-853, 2013.

13. IAEA - International Atomic Energy Agency. Management of Disused Sealed Radioactive Sources. Nuclear Energy Series NW- T-1.3. Vienna, 2014. 165p. 
14. RABSKI, H.; PYNE, J. Evolution of an Orphaned Source Management Strategy. In: Safety and Security of Radioactive Sources: Maintaining Continuous Global Control of Sources Throughout Their Life Cycle, Abu Dhabi, 2013. p. 325-332.

15. VAN DER MEERSCH, D.; DE WILDE, K. Belgian Global Approach on the Radiological Surveillance of Radioactive Orphan Sources and Radioactive Substances In Scrap Metal And Non-Radioactive Waste. In: Safety and Security of Radioactive Sources: Maintaining Continuous Global Control of Sources Throughout Their Life Cycle, Abu Dhabi, 2013. p. 307-324.

16. JAMMAL, R.; RÉGIMBALD, A. Successes and Challenges in Implementing Sustainable Approaches in Canada to Strengthen the Safety and Security of Radioactive Sources. In: Safety and Security of Radioactive Sources: Maintaining Continuous Global Control of Sources Throughout Their Life Cycle, Abu Dhabi, 2013. pp. 129-138. 